\title{
Endoprótesis biliar en el manejo transitorio de la coledocolitiasis
}

\author{
Mario Anselmi $M^{1,2}$, Juan Carlos Acuña $C^{1}$, \\ Ana Del Valle $M^{2}$, Ana María $G$ emmato $P^{2}$. \\ Endoscopic biliary stents \\ for the temporary management \\ of choledocholithiasis
}

Background: Endoscopic extraction of biliary tract stones is safe and effective. When the procedure is not successful, the use of a temporary stent can be a solution. Aim: To prospectively analyze the usefulness of endoscopic biliary stents in the temporary management of biliary obstruction due to choledocholithiasis. Material and methods: Analysis of 51 consecutive patients (age range 21-88 years, 34 females) with common bile duct stones that, from January 1999 to December 2001, were subjected to an endoscopic insertion of a biliary stent. Results: The indications for stent placement were a large stone in 40 patients (78\%), the insecurity of a complete biliary tract cleaning in eight (16\%) and technical difficulties in three (6\%). Twenty seven patients (52.9\%) were jaundiced and 17 (33.3\%) had cholangitis. The prostheses remained in place until definitive resolution of the choledocholithiasis in 47 patients (92\%) and migrated in 4 (8\%). Bilirubin levels became normal in all cases with jaundice and infection resolved in all those with cholangitis. The definitive treatment of choledocholithiasis was done endoscopically in 28 patients (58\%) and surgically in 20 (42\%). Three patients were lost from follow up. Of these, one patient (2\%) died 14 months later due to a recurrent cholangitis. The remaining two patients were asymptomatic and with the prostheses still in place 522 and 560 days post stent placement. Conclusions: When the immediate endoscopic resolution of choledocholithiasis is not possible, temporary stenting is a simple and safe therapeutic alternative that allows patients to be free of obstructive complications until the definitive treatment is carried out (Rev Méd Chile 2006; 134: 721-5).

(Key words: Cholangitis; Gallstones, common bile duct; Stents)

Recibido el 10 de junio, 2005. Aceptado el 24 de diciembre, 2005.

${ }^{1}$ Departamento de Cirugía, Facultad de Medicina, Universidad de Concepción. ${ }^{2}$ Unidad de Cirugía Endoscópica, Servicio de Cirugía, Hospital Guillermo Grant Benavente, Concepción, Chile.

L a extracción endoscópica de los cálculos de la vía biliar, se ha convertido en un método seguro, efectivo y ha alcanzado un éxito superior

Correspondencia a: Dr. Mario Anselmi M. Departamento de Cirugía, Facultad de Medicina, Universidad de Concepción, Chile. Fono Fax: 56-41-204881. E mail: anselmi@entelchile.net a $90 \%{ }^{1-3}$. Sin embargo, hay casos en que, a pesar de una esfinterotomía adecuada, la vía endoscópica debe descartarse o diferirse, cuando debido al gran tamaño del cálculo no puede extraerse en una primera oportunidad o cuando quedan dudas respecto a la limpieza del hepatocolédoco.

La persistencia de litiasis en vía biliar implica la posibilidad de obstrucción, colangitis y pancreati- 
tis $^{3,4}$. Se ha comprobado un aumento estadísticamente significativo en el riesgo de este tipo de morbilidad en los pacientes con persistencia de cálculos, apareciendo complicaciones mayores hasta en $32,3 \%$ de los casos $^{5}$. Con el objeto de prevenir estas complicaciones, es necesario asegurar un drenaje adecuado de la vía biliar, lo que endoscópicamente puede lograrse mediante la inserción de una sonda nasobiliar o de una prótesis. La sonda nasobiliar comunica la vía biliar con el exterior. De esta manera da un acceso permanente a ella, permitiendo, entre otras cosas, no sólo descomprimirla e instilar soluciones, sino que también efectuar colangiografías seriadas.

La inserción de una prótesis, al prevenir la impactación de un cálculo en la ampolla de Vater, mantiene la permeabilidad de la vía biliar. Puede, por lo tanto, representar una alternativa al uso de una sonda nasobiliar mientras el problema se soluciona en forma definitiva ${ }^{6}$.

El objetivo de este trabajo es analizar, en forma prospectiva, la utilidad de las prótesis biliares instaladas por vía endoscópica en el manejo transitorio de la obstrucción biliar, debida a coledocolitiasis.

\section{MATERIAL Y MÉTODO}

Entre enero de 1999 y diciembre de 2001, 932 pacientes portadores de una coledocolitiasis, comprobada mediante colangiopancreatografía endoscópica, fueron sometidos a un intento de extracción endoscópica de los cálculos de la vía biliar. Del total, 473 (50,8\%) eran colecistectomizados y $459(49,2 \%)$ tenían la vesícula in situ. A 51 pacientes $(5,5 \%)$, cuya coledocolitiasis no pudo ser solucionada endoscópicamente, se les sometió a la inserción de una prótesis biliar con el fin de asegurar la permeabilidad de la vía biliar hasta la solución definitiva del problema.

La edad de los pacientes fluctuó entre los $21 \mathrm{y}$ 88 años (promedio: 64,1 1 19,2), $17(33,3 \%)$ eran hombres y $34(66,7 \%)$ mujeres. En 40 casos $(78,4 \%)$, la indicación para la inserción de la prótesis fue la presencia de un cálculo grande, en $8(15,6 \%)$ la inseguridad de limpieza de la vía biliar y en $3(5,8 \%)$ dificultades técnicas en el procedimiento. Del total, 27 pacientes $(52,9 \%)$ presentaban ictericia y 17 tenían colangitis
(33,3\%). Todos los procedimientos fueron efectuados con el paciente hospitalizado y por uno de los autores. La técnica de insención fue la clásica ${ }^{7,8}$, introduciendo prótesis rectas $7 \mathrm{Fr}$ a través de un duodenoscopio con un canal de trabajo de 3,2 $\mathrm{mm}$. No se utilizó antibióticos, salvo en pacientes con sospecha de colangitis.

En aquellos en que se consideró imposible extracción endoscópica inmediata, después de la esfinterotomía, se instaló una prótesis. En los otros, se intentó la remoción mediante canastillos de dormia y/o litotriptor, de tantos cálculos como fuera posible y en caso de duda de limpieza de la vía biliar, se procedió a insertar una endoprótesis. Finalizado el procedimiento, los pacientes continuaron en observación para el control de su evolución precoz. Permanecieron hospitalizados aquellos que su condición clínica lo aconsejaba 0 en los que se esperaba una solución definitiva quirúrgica o endoscópica rápida. Los demás fueron dados de alta con un mínimo de $24 \mathrm{~h}$ de observación.

Los enfermos con prótesis in situ fueron controlados clínica y radiológicamente en consultorio externo hasta la solución definitiva del problema.

\section{RESULTADOS}

En 49 pacientes $(96,1 \%)$ la instalación fue exitosa al primer intento y en 2 enfermos $(3,9 \%)$ debió realizarse un segundo intento debido a sangrado por precorte. Un paciente $(1,9 \%)$ presentó una pancreatitis aguda necrohemorrágica Balthazar E, como complicación derivada del procedimiento, recuperándose en forma satisfactoria.

La prótesis permaneció en posición, hasta la resolución definitiva de la coledocolitiasis en 47 pacientes (92,1\%) y migró en 4 casos (7,8\%). Los valores de bilirrubina se normalizaron en todos los pacientes con ictericia y el cuadro séptico cedió en la totalidad de los enfermos con colangitis.

La permanencia promedio de las prótesis fue de 157,8 999,6 días (rango: 3-602 días). Sin embargo, en 35 pacientes $(68,6 \%)$, la limpieza de la vía biliar se efectuó antes de 30 días de instalada la prótesis y en $6(11,8 \%)$ antes de 60 días. De los 10 restantes $(19,6 \%)$ en $4(7,8 \%)$ la coledocolitiasis se solucionó antes de tres meses y en $3(3,9 \%)$ la 
prótesis se retiró durante el acto quirúrgico a los 245, 360 y 425 días por sucesivas postergaciones de su cirugía.

En 48 pacientes, el tratamiento definitivo de la coledocolitiasis fue a través de un nuevo procedimiento endoscópico en $28(58,3 \%)$ y mediante cirugía en 20 (41,7\%). De estos últimos, en 13 (65\%) se indicó manejo quirúrgico de la coledocolitiasis, debido a que el gran tamaño del cálculo impedía la captura con los canastillos disponibles en esa época. En 4 pacientes los cálculos estaban ubicados en serie, ocupando toda la vía biliar extrahepática por lo que no era posible abrir los canastillos para atraparlos y extraerlos. Dos casos eran portadores de un síndrome de Mirizzi y uno presentaba una severa disrelación entre el tamaño del cálculo y el de la vía biliar distal. Tres enfermos no volvieron a control. De éstos, al término del seguimiento uno (2\%) había fallecido 14 meses después de una colangitis recidivada. En los 2 restantes, la prótesis aún permanecía in situ y estaban asintomáticos 522 y 560 días post inserción.

\section{DisCUSIÓN}

Los procedimientos de cirugía endoscópica han causado un enorme impacto en el tratamiento de la coledocolitiasis. Aunque la principal indicación para el manejo endoscópico de la litiasis biliar sigue siendo la presencia de cálculos residuales 0 recidivantes, cada vez con mayor frecuencia se utiliza en pacientes con vesícula in situ, ya sea como paso preoperatorio o en casos seleccionados, como tratamiento definitivo 9,10 .

Es claro que en pacientes portadores de una coledocolitiasis post colecistectomía, la extracción endoscópica de los cálculos parece ser más simple, efectiva y barata que la exploración quirúngica $1,3,9,11$

Uno de los problemas asociados al método endoscópico para la extracción de cálculos de la vía biliar, y que está fuera de control del operador, es la retención de éstos. En efecto, durante el primer intento, sólo se logra la remoción completa de los cálculos de la vía biliar en $73 \%$ de los pacientes $^{12}$. En ocasiones, el riesgo de dejar un cálculo retenido es de importancia secundaria comparado con la urgencia de descomprimir la vía biliar, como por ejemplo en pacientes con colangitis $^{13,14}$. En otras, condiciones locales tales como divertículos yuxtapapilares o estenosis biliar proximal al cálculo pueden imposibilitar la extracción de éste, a pesar de una esfinterotomía adecuada $^{15}$. Por último, la forma, el tamaño o la presencia de una panlitiasis coledociana, son factores que impiden la extracción endoscópica del cálculo o crean inseguridad respecto a la limpieza de la vía biliar ${ }^{12}$.

La persistencia de una litiasis biliar, posterior a la manipulación endoscópica, se asocia a un aumento estadísticamente significativo en el riesgo de morbilidad post-procedimiento, apareciendo complicaciones mayores en aproximadamente un tercio de los $\operatorname{casos}^{5}$. Con el objeto de prevenir esta situación, es necesario asegurar un drenaje adecuado de la vía biliar, lo que endoscópicamente puede lograrse mediante la inserción de una sonda nasobiliar o de una prótesis. La sonda nasobiliar fue creada inicialmente para el manejo transitorio y por corto plazo de una obstrucción biliar. Entre sus ventajas se cuenta el hecho que permite controlar las características y el débito diario de bilis, hace posible efectuar colangiografías de control 0 instilación de antibióticos 0 sustancias destinadas a disolver los cálculos ${ }^{16,17}$. Sin embargo, son caras para nuestro medio, no siempre son bien toleradas por los enfermos, pueden obstruirse, debe permanecer hospitalizado.

La inserción de una endoprótesis, es un procedimiento cada vez más utilizado para descomprimir la vía biliar. Debido a su simplicidad y eficacia, este método ha tenido una gran aceptación y actualmente representa un recurso fundamental en la endoscopia biliopancreática. Aún más, sus indicaciones se han ampliado enormemente, constituyendo hoy día no solo una alternativa a la terapia quirúrgica en el manejo de la obstrucción biliar maligna ${ }^{18}$, sino que además se indican en una variedad de patologías benignas litiásicas y no litiásicas $9,19,20$. En los cuadros obstructivos neoplásicos, la prótesis provee el lumen para el drenaje biliar. Dejadas in situ por largo tiempo, tienden a obstruirse, por lo que el manejo de estos pacientes presupone su cambio, el que se hará con mayor o menor frecuencia, dependiendo de una serie de factores incluyendo la amplitud de su lumen ${ }^{21,22}$. 
En cambio, en una coledocolitiasis no resuelta por vía endoscópica, la inserción de una prótesis impide la impactación del cálculo en la ampolla de Vater y de esta manera mantiene la permeabilidad de la vía biliar. Por este motivo, su obstrucción no es importante y los cambios son innecesarios ${ }^{15,23}$. En efecto, uno de nuestros pacientes mantuvo la prótesis in situ por 560 días sin presentar complicaciones derivadas de una obstrucción calculosa. Por la misma razón, éstas son utilizadas como alternativa al manejo quirúrgico de la coledocolitiasis en pacientes geriátricos de alto riesgo $0^{4,24,25}$.

Con equipo adecuado y personal entrenado, la inserción de una endoprótesis es un procedimiento seguro y relativamente simple. Como lo demuestran los resultados de nuestra y otras series ${ }^{7}$, completada la esfinterotomía, la endoprótesis puede ser instalada al primer intento en casi todos los pacientes. La esfinterotomía facilita el drenaje de la bilis, mientras que la prótesis mantiene al cálculo en el conducto proximal, previniendo su impactación distal (Figura 1) y sirve de brecha alrededor de la cual la bilis puede fluir hacia el duodeno ${ }^{26}$. En nuestra serie, la bilirrubinemia permaneció dentro de límites normales en la totalidad de los pacientes con prótesis in situ, sin que al momento de la resolución definitiva de la coledocolitiasis se presentaran complicaciones derivadas de ésta. Asimismo, en pacientes con colangitis concomitante, el cuadro séptico regresó en todos los casos que la presentaban. Una vez drenada la vía biliar, el enfermo puede ser controlado en forma ambulatoria y la coledocolitiasis solucionada posteriormente en condiciones óptimas.

La migración de la prótesis fue el único evento adverso observado con este tipo de tratamiento. Esta complicación, que se presenta en alrededor de $6 \%$ de los casos parece ser más frecuente en pacientes con marcada dilatación de los conductos ${ }^{27,28}$. Los movimientos de los cálculos y las esfinterotomías amplias pueden facilitar la salida hacia el duodeno. En nuestra serie, la prótesis permaneció en posición hasta la resolución definitiva de la patología litiásica en 47/51 pacientes $(92,1 \%)$. Es imposible predecir qué prótesis migrará y cuál no. Sin embargo, probablemente el uso de prótesis rectas favorezca la migración y el de prótesis tipo epigtail»la prevenga ${ }^{15,29}$.
La única complicación derivada del procedimiento fue una pancreatitis aguda necrohemorrágica Balthazar E, la que evolucionó satisfactoriamente. Sin embargo, como se sabe, ésta es una complicación de la colangiopancreatografía retrógrada más que de la inserción de la endoprótesis misma.

Los resultados de este trabajo sugieren que frente a una coledocolitiasis cuya resolución inmediata por vía endoscópica no es posible, la instalación de una prótesis es una alternativa terapéutica simple y segura, que permite mantener a los pacientes libres de complicaciones obstructivas hasta la solución definitiva del problema por vía endoscópica o quirúrgica.

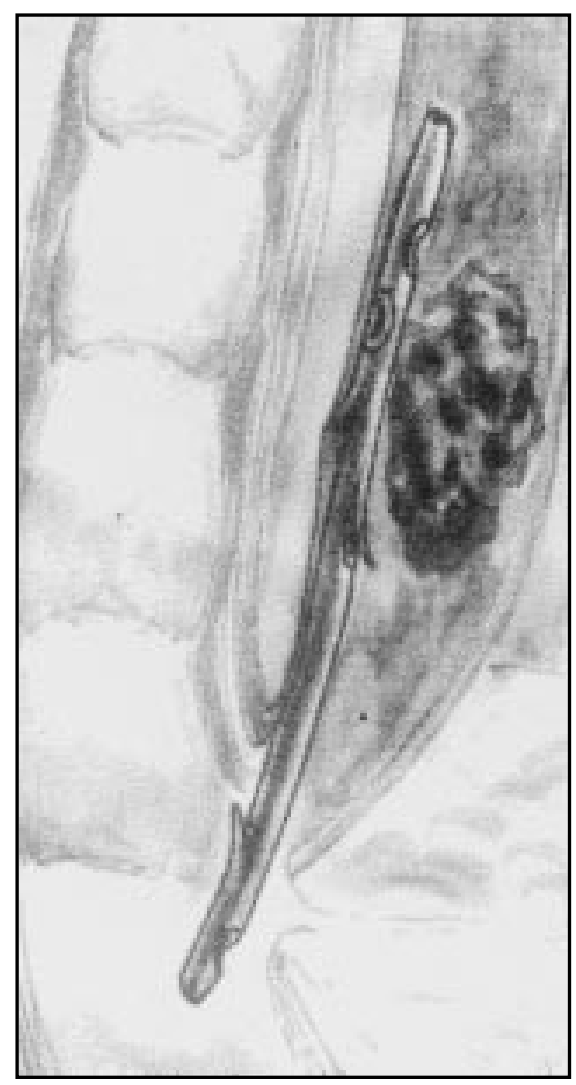

Figura 1. La prótesis mantiene al cálculo retenido en el conducto proximal previniendo su impactación distal y sirve de brecha alrededor de la cual la bilis puede fluir al duodeno. 


\section{REFERENCIAS}

1. Соттол P. Endoscopic Management of bile duct stones (apples and oranges). Gut 1984; 25: 587-97.

2. Vaira D, Dànna L, Ainley C, Dowsett J, Wimams S, BAIUE J ET ALS. Endoscopic sphicterotomy in 1000 patients. Lancet 1989; ii (8660): 431-4.

3. ANSELMI M, Silva I, InNoCenti F. Coledocolitiasis post colecistectomía: Esfinterotomía vs reoperación. Rev Chil Cir 1997; 49: 188-92.

4. McNulty JG, Hickery N, Thornton J, Osborne DH. Percutaneous placement of biliary stents for the treatment of high risk patients with jaundice due to common bile duct stones. Am J Gastroenterol 1999; 94: 480-3.

5. Neoptolemos JP, Davison BR, Shaw DE, Loyd D, CarrLOCKE DL, FOSSARD DP. Study of common bile duct exploration and endoscopic sphincterotomy in consecutive series of 438 patients. Br J Surg 1987; 74: 916-21.

6. Hochberger J, TeX S, Maiss J, Hahn EG. Management of difficult common bile duct stones. Gastrointest Endosc Clin North Am 2003; 13: 623-34.

7. Соттоn P. Endoscopic methods for relief of malignant obstructive jaundice. World J Surg 1984; 8: 854-61.

8. SoEHENDRA N, GRIMm H. Endoscopic retrograde drainage for bile duct cancer. World J Surg 1988; 12: 85-90.

9. Bowrey DJ, Fugeistone LJ, Solomon A, Thomas G, Shandall AA. Common bile duct stenting choledocholithiasis: a district general hospital experience. Postgrad Med J 1998; 74: 358-60.

10. Schreurs WH, Vies WJ, Stuifbergen WH, Oostvogel HJ. Endoscopic management of common bile duct stones leaving the gallbladder in situ. A cohort study with long-term follow-up. Dig Surg 2004; 21: 60-4.

11. GhaZi A, WASHINGTon M. Endoscopic Retrograde Cholangio pancreatography, Endoscopic Sphincterotomy, and Biliary Drainage. Surg Clin North Am 1989; 69: 1249-74.

12. García-Cano Liscano J, Gonzáiez Martín Ja, Pérez Sola A, Morilas Arino MJ. Success rate of complete extraction of common bile duct stones at first endoscopy attempt. Rev Esp Enferm Dig 2002; 94: 340-50.

13. Anseimi M, Salgado J, Arancibia A, Awi C. Colangitis aguda debida a coledocolitiasis. Cirugía tradicional 0 drenaje biliar endoscópico. Rev Méd Chile 2001; 129: 757-62.

14. Bornman PC, van Beijon JI, Krige JE. Management of cholangitis. J Hepatobiliary Pancreat Surg 2003: 10: 406-14.

15. MaXton DG, TweEDie DE, Martin DF. Retained common bile duct stones after endoscopic sphinctero- tomy: temporary and long term treatment with biliary stenting. Gut 1995; 36: 446-9.

16. Johnson GK, Geenen JE, Venu RP, Schmalz MJU, Hogan WJ. Treatment of non-extractable common bile duct stones with combination ursodeoxycholic acid plus endoprotheses. Gastrointest Endosc 1993; 39: 528-31.

17. MaXton DG, TweEde DE, MaRTIN DF. Stenting for choledocholithiasis; temporizing or therapeutic?Am J Gastroenterol 1996; 91: 615-6.

18. Shepherd HA, Royie G, Ross AP, Diba A, Arthur M, COLN-Jones D. Endoscopic biliary endoprostheses in the palliation of malignant obstruction of the distal common bile duct: a randomized trial. Br J Surg 1988; 75: 1166-8.

19. Costamagna G, Shah SK, Tringall A. Cument management of postoperative complications and benign biliary strictures. Gastrointest Endosc Clin North Am 2003; 13: 635-48.

20. Katsinelos P, Paroutoglou G, Beltsis A, Tsolkas $P$, ArvanItI M, KatSIBA D ET AL. Endobiliary endoprosthesis without sphincterotomy for the treatment of biliary leakage. Surg Endosc 2004; 18: 165-6.

21. Coene PP, Groen AK, Cheng J, Out MM, Tytgat GN, HunBREGTSE K. Clogging of biliary endoprosthesis: a new perspective. Gut 1990; 31: 913-7.

22. Speer A, Сotton P, MacRae K. Endoscopic management of malignant biliary obstruction: stents of $10 \mathrm{Fr}$ gauge are preferable to stents 8 French gauge. Gastrointest Endosc 1988; 34: 334: 412-7.

23. BUTENSKY MS. Long-term stenting for choledocholithiasis. Conn Med 2000; 64: 413-4.

24. Bergmann JJ, Rauws EA, Tijssen JG, Tytgat GN, HuIBREGTSE K. Biliary endoprostheses with endoscopically erretrievable common bile duct stones; report on 177 patients. Gastrointest Endosc 1995; 42: 272-3.

25. Sugirama M, Атом Y. Endoscopic sphicterotomy for bile duct stones in patients 90 years of age and older. Gastrointest Endosc 2000; 52: 187-91.

26. Zimmons DS, CiemetT AR. Endoscopic stents and drains in the management of pancreatic and bile duct obstruction. Surg Clin Noth Am 1982; 62: 837-44.

27. Foutch P, Hardan J, Sanowski R. Endoscopic placement of biliary stents for treatment of high risk geriatric patients with common bile ducts stones. Am J Gastroenterol 1989; 84: 527-9.

28. Johanson JF, Schmaiz MT, GREenEN JE. Incidence and risk factors for biliary and pancreatic stent migration. Gastrointest Endosc 1992; 38: 341-6.

29. Cotton PB, Forbes A, Leung JW, Dineen L Endoscopic stenting for long-term treatment of large bile duct stones: 2 to 5 years follow up. Gastrointest Endosc 1987; 33: 411-22 